\title{
SYNERGISTIC USE OF IMAGER WINDOW OBSERVATIONS FOR CLOUD- CLEARING OF SOUNDER OBSERVATION FOR INSAT-3D
}

\author{
Jyotirmayee Satapathy*, P.K. Thapliyal, M.V. Shukla, C. M. Kishtawal \\ Atmospheric and Oceanic Sciences Group, Space Application Centre, Ahmedabad-380015, India \\ *E-mail: jsatapathy84@gmail.com
}

\begin{abstract}
:
The retrieval of atmospheric temperature and water vapor profiles from infrared Sounder are severely limited by the presence of cloud. Therefore, retrieval from infrared sounding observations is performed only over clear-sky atmospheric conditions. The probability of finding a clear-sky pixel at spatial resolution of $10 \mathrm{~km}$ is found to be very small globally. This study presents a quantitative analysis of the clear-sky probability that is carried out for different months over the Indian region for INSAT-3D Sounder. The probability of a clear-sky is found to be $\sim 7 \%$ for the field of view of $10 \mathrm{~km}$ corresponding to the INSAT-3D Sounder. This statistical analysis is established using MODIS cloud mask having 95\% confidence level at $1 \mathrm{~km}$ resolution spread in the region between $50 \mathrm{E}-110 \mathrm{E}$ and $30 \mathrm{~S}-30 \mathrm{~N}$. This necessitates cloud clearing to remove the effect of partial clouds in the Sounder FOV to provide a clear-sky equivalent sounding retrieval.

Various methods were explored to derive the cloud-cleared radiances using supplementary information such as high resolution infrared or microwave observations. This study presents an effort to use the existing traditional method to derive optimal cloudcleared radiances for INSAT-3D Sounder, by estimating the fractional cloud cover using collocated high resolution INSAT-3D Imager window channel observation. The final Sounder cloud-cleared radiances have been validated with the operational AIRS L2 cloud-cleared radiance products.

Nevertheless, the statistical analysis of clear-sky probability over Indian region also provides a significant insight towards the dependency of spatial resolution and the considerable field-of-regard (FOR) in obtaining the clear-sky area in the satellite observations. This, in a way, necessitates the cloud-clearing for coarser resolution sensors and at the same time, states the benefits of using very high resolution sensors. It has been observed that FOV of $1 \mathrm{~km}$ and by choosing a reasonably good FOR can eliminate the cloudy-sky hindrances by increasing the probability of clear-sky from 5\% to $50 \%$.
\end{abstract}

Keywords: Cloud-clearing, INSAT-3D, Sounder, Imager, Clear-sky Probability

\section{INTRODUCTION}

Remote sensing and meteorological studies are always associated with the fact whether the sky is clear or cloud contaminated, because cloud influences the determinism of the results and hinders in the sounding and retrieval processes. Although cloud is the most important part of the atmospheric studies, it provides adversities in the path of remote sensing particularly in infrared part of the electromagnetic spectrum. Hence, there is always a necessity to have an estimate of the cloud coverage globally or so. But clouds are not distributed uniformly in a in various parts of the globe. Due to the dominance of the convective processes cloud formation and presence is highly probable with a large uncertainty in the tropical region.

Indian region being in the vicinity of the tropics, it is mostly occupied with clouds. In this study the annual clearsky probabilities over Indian region at different satellite sensor footprints have been estimated, which lays the foundation to the necessity of cloud-clearing technique that can enhance the sounding retrieval yield for weather, climate and environmental analysis over this region. This work has been carried out by considering three different field-of-views (FOVs) within three different field-ofregards (FORs) over the Indian region using Moderate resolution Imaging Spectro-radiometer (MODIS) operational cloud masks at $95 \%$ confidence level. Annual average is taken from monthly averages representing four different seasons.

Apart from that, the essentiality of the clear-sky as well as the clear radiance is quite obvious for meteorological observations which leads to ample researches to undergo towards developing cloud-clearing technique [1-2]. First, most of the research was concentrated on the problem of retrieving the temperature and humidity profiles from the cloud contaminated radiances and second is to deduce the cloud effect on geophysical parameter observations. But these results still present the ambiguity and limitation which ultimately demand the cloud clear products for weather applications [3].

The cloud-clearing has been achieved in several ways in the past. Out of these, the traditional single band approach [2] that makes use of the high resolution observations to attempt cloud-clearing of a coarser resolution observation is a most effective approach. In the present study, this method is attempted by collocating high resolution INSAT3D Imager observations inside INSAT-3D Sounder observation synergistically by considering the clear-sky radiance observed from Imager which is further used to derive cloud-cleared radiance for the Sounder FOVs. The derived cloud-cleared radiances are validated with that of AIRS L2 operational cloud-cleared products. Along with 
this, a sensitivity study to determine the impact of the partial cloud cover on the Sounder observation has also been carried out.

This kind of work has not been attempted earlier for Indian satellite observations. Therefore, it carries significance for sounding observations under partially cloudy sky conditions to increase the yield for atmospheric profiles retrieval as well as for the data assimilation in NWP models.

\section{DETERMINATION OF CLEAR SKY PROBABILITY:}

This work is carried with cloud masks taken from MODIS L2 products from Terra as well as Aqua satellites of NASA-EOS (Earth Observation Satellite). MODIS cloud masks are obtained using various cloud detection algorithms taken together. It makes use of 31 bands of 5visible and broad spectrum of infrared wavelengths which makes this cloud masking accurate and accepted globally. These cloud masks are divided into 4 categories, viz. cloudy, clear, probably clear and partially cloudy. Out of these the clear flags are considered to be $95 \%$ probable clear observations. These clear pixels are considered for this experiment to identify and provide quantitative estimate of the clear regions [4].

MODIS make high spatial resolution $(1 \mathrm{~km})$ observations from polar sun-synchronous orbit with equatorial crossing time of 01:30 AM/PM (local time) in ascending/descending orbit. For each day maximum 5 passes of its swath are obtained in the INSAT-3D Imager scan region and are considered for the study.

Most of the parameters retrieval is carried out over a larger area known as the Field-of-Regard (FOR) to reduce the sensor noise by averaging these observations and also to reduce the possibility of cloud contamination. We have examined three different FOR with sizes of 10, 30 and 50 $\mathrm{km}$ (corresponding to single pixel, $3 \times 3$ and $5 \times 5$ pixel FOR, respectively) for FOVs of various sizes 2,4 and $10 \mathrm{~km}$ corresponding to the spatial resolution of the sensor. The probability has been computed for a minimum number $(n)$ of clear FOVs within each FOR. The number $n$ varies as 1 , $2,4,8,16,32$ and all. The clear FOV is identified by using MODIS clear pixels inside every FOV at 3 different confidence levels as $100 \%, 75 \%$ and $50 \%$. This method has been applied for entire collocated dataset at 3-day interval and a monthly average is computed by averaging these observations. This is carried out for four different months (January, April, July and October) representing different seasons in the year. Finally combined average is taken from these 4 monthly averages to calculate the annual average of the clear-sky probability over Indian region.

The annual probability of clear sky is shown in the figure 1 . It can be seen from the figure that as the FOV decreases the probability of clear sky increases. FOV is an analogy to the spatial resolution of the instrument (Sounder/Imager). Hence it can be seen that the probability of clear sky becomes more than double to that of $10 \mathrm{Km}$ resolution for each case. Similarly, as the FOR size is increased, the probability increases for each case of FOVs by $20-30 \%$. the Indian satellites as the cloud-cleared radiances corresponding to partially cloudy pixels can enable the retrieval of surface and atmospheric parameters. Besides, the novelty lies in the weather prediction aspect where no microwave sounding is available. This improvement will have potential application for the operational processing of Also with the decreased confidence level of cloud flag of MODIS pixels the probability has been increased by $5-10 \%$ due to obvious reasons.

The significant increment of probability with a lower FOV such as $2 \mathrm{~km}$ leads to the conclusion that with the finer resolution it is possible to find clear pixels for retrieval and does away with the need to do cloud-clearing. Contrary to this, for the FOV of $10 \mathrm{~km}$ the cloud-clearing becomes a necessary feature due to smaller probability of finding clear-pixel. It also explains the fact that considering larger size of FOR, e.g. $5 \times 5$ pixels, gives an advantage as the probability for finding clear pixel is higher in larger FOR than the individual pixel. In addition to this, it will be advantageous to have high resolution observations (e.g. Imager) corresponding to the coarser resolution observations (e.g. Sounder) that will lead to the cloudclearing of later.

\section{SENSITIVITY STUDY TO EXAMINE THE IMPACT OF FRACTIONAL CLOUD COVER:}

The uncertainty in the observed brightness temperature due to partial cloud cover within a FOV is computed and presented in the figure- 2 for considering different cloud fractions with cloud top temperatures of $200 \mathrm{~K}, 250 \mathrm{~K}$ and $275 \mathrm{~K}$. Here we have considered the clear scene having brightness temperature of $300 \mathrm{~K}$ as a part of the sensitivity study. The basic equation considered for this theoretical analysis is given by:

$R=\alpha \cdot R_{\mathrm{cld}}+(1-\alpha) \cdot R_{\mathrm{clr}}$

Where, $R$ is total radiance of the cloud contaminated scene, $R_{\text {cld }}$ is the cloudy-sky radiance calculated for different cloud top, $R_{\mathrm{clr}}$ is the clear-sky radiance for the scene brightness temperature of $300 \mathrm{~K}$, and $\alpha$ is the cloud fraction assuming the cloud emissivity to be unity. These radiances are computed using Planck's formula corresponding to the cloud top temperature and surface temperature.

From computed satellite radiance $(R)$ again the brightness temperature corresponding to the cloud contaminated scene is calculated. The inter conversion of brightness temperature and radiance is carried using Planck's formula. The results are shown in figure 2 .

From figure 2, it can be seen that for cloud fraction of approximately $2 \%$ in the footprint brings down its brightness temperature by $1 \mathrm{~K}$ for very cold or high cloud with cloud top temperature $200 \mathrm{~K}$, whereas for moderately cold or mid-level clouds with cloud top temperature $225 \mathrm{~K}$, the change of $1 \mathrm{~K}$ in the brightness temperature is obtained with $3 \%$ of cloud fraction and for warm or low level clouds with cloud top temperature $250 \mathrm{~K}$, this is $5 \%$. This study ensures the sensitivity for Sounders in terms of the change in brightness temperature by the presence of cloud. This indicates that contamination of $2 \%$ high cloud, or $3 \%$ of mid-level cloud, or $5 \%$ of the low-level cloud will lead to 
an uncertainty of about $1 \mathrm{~K}$ in the brightness temperature and the same will adversely affect the accuracy of the sounding products. These levels of cloud contamination are very difficult to detect using conventional cloud detection algorithm. Therefore, the treatment of these partial clouds in the coarser resolution sounders needs to be attempted before actual retrieval is performed.
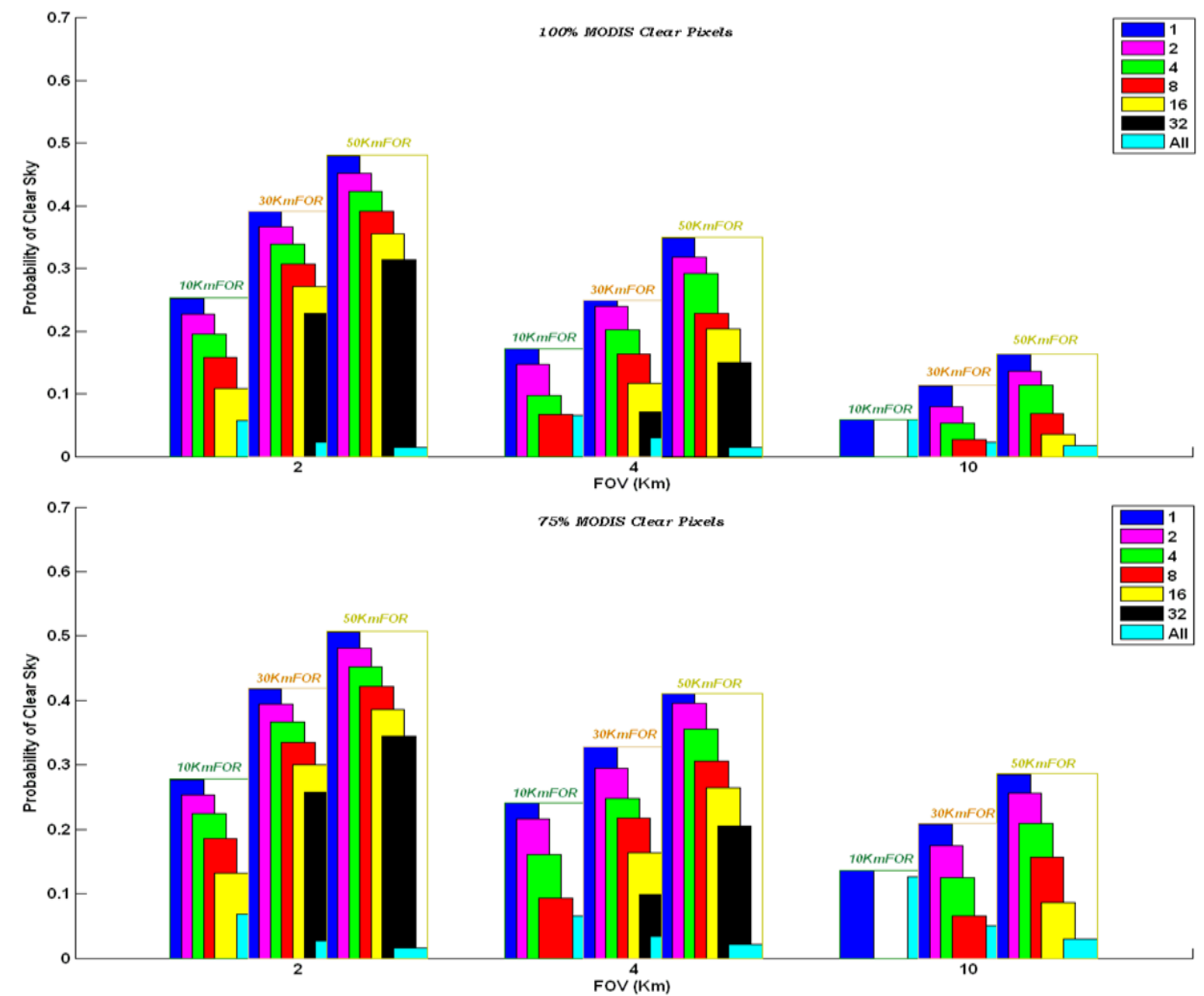

75\% MODIS Clear Plxels
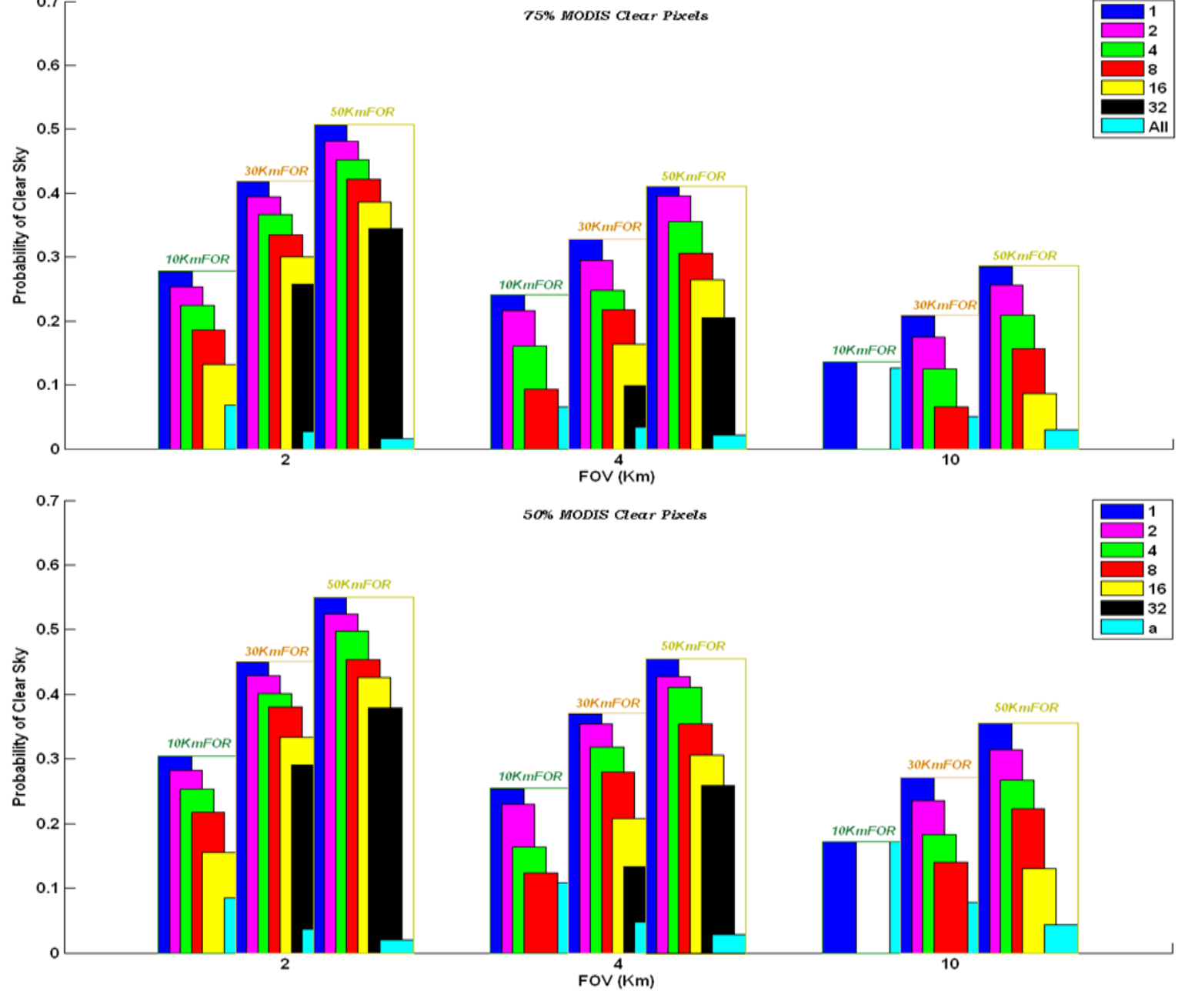

Figure 1. Annual Probability of clear sky over Indian region 

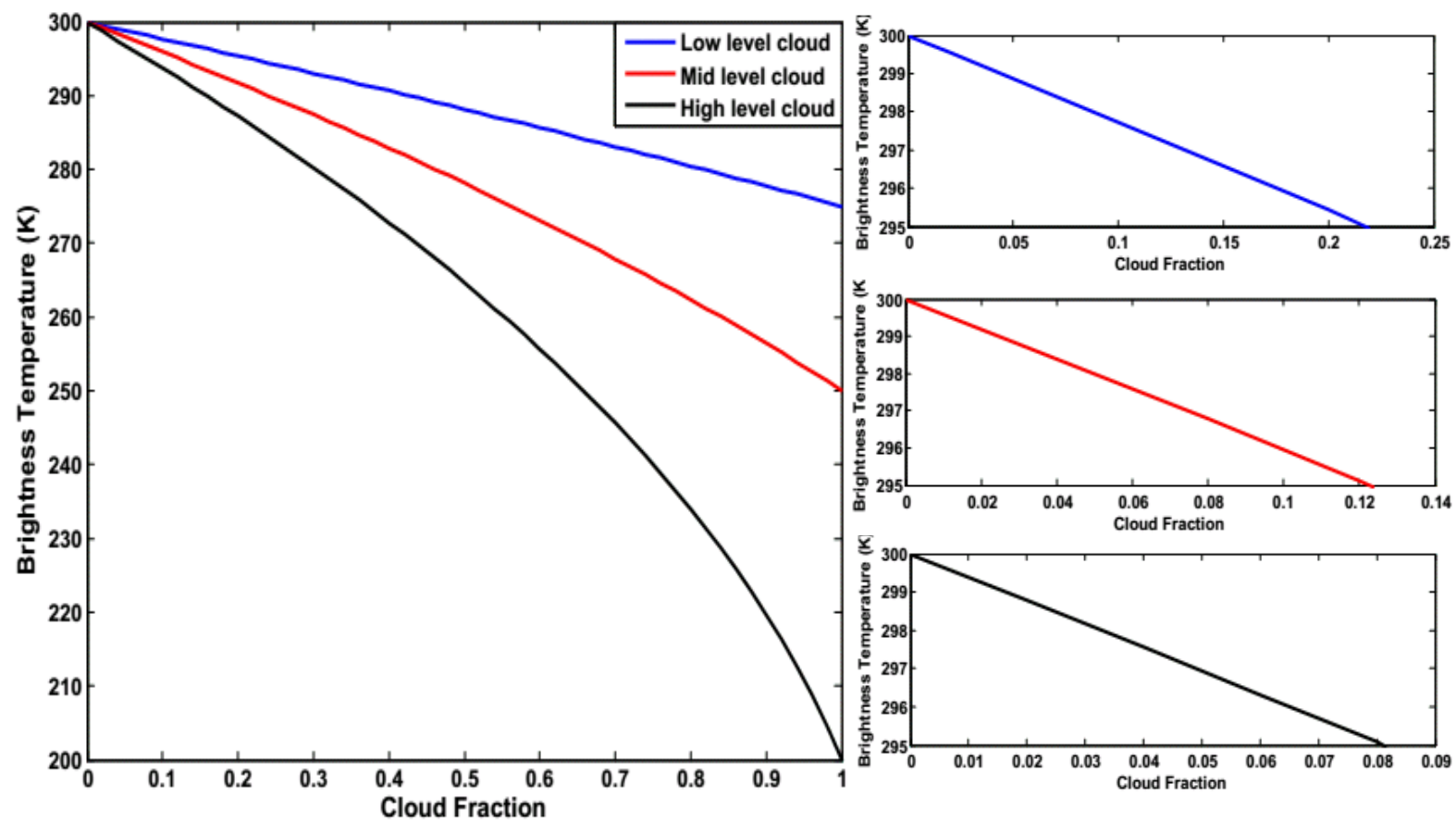

Figure 2: Variation of Brightness temperature with cloud fractions for different types of cloud

\section{CLOUD CLEARING METHODOLOGY:}

As shown in the figure-3, if $R_{1}$ and $R_{2}$ are radiances of two adjacent INSAT-3D Sounder FORs for the window channel with cloud fractional coverage $n_{1}$ and $n_{2}$ and emissivity of $\varepsilon_{1} \& \varepsilon_{2}$ respectively, then the cloudy radiances of the two adjacent pixels can be expressed as [5]

$$
\begin{aligned}
& R_{1}=\left(1-n_{1} \varepsilon_{l}\right) \tilde{R}_{\text {clear }}+n_{1} \varepsilon_{1} R_{\text {cloudy }} \\
& R_{2}=\left(1-n_{2} \varepsilon_{2}\right) \tilde{R}_{\text {clear }}+n_{2} \varepsilon_{2} R_{\text {cloudy }}
\end{aligned}
$$

Where $\tilde{R}_{\text {clear }}=$ Clear radiance of the pixel

$$
\mathrm{R}_{\text {cloudy }}=\text { Radiance overcastted by cloud }
$$

$N^{*}$ can be calculated using either of the following equations that is obtained by rearranging equation (2) and (3) as;

$$
\begin{aligned}
& \frac{\mathrm{R}_{1}-\tilde{R}_{\text {clear }}}{\mathrm{R}_{2}-\tilde{R}_{\text {clear }}}=\mathrm{N}^{*} \\
& \frac{\mathrm{n}_{1} \varepsilon_{1}}{\mathrm{n}_{2} \varepsilon_{2}}=\mathrm{N}^{*}
\end{aligned}
$$

After estimating $\mathrm{N}^{*}$ from any of the above equation, cloud clear radiance, $R_{\text {clear }}$ for each INSAT-3D Sounder pixel can be retrieved as given in the following equation:

$$
R_{\text {clear }}=\frac{R_{1}-N^{*} \times R_{2}}{1-N^{*}}
$$

For computation of $N^{*}$ in equation (4) the clear sky radiance, $\tilde{R}_{\text {clear }}$ can be determined traditionally by considering the collocated microwave observation or high resolution IR observations. Here, the later method is employed by considering collocated high resolution INSAT-3D Imager observations inside the INSAT-3D
Sounder FOR. We have considered average radiance within FOR of $3 \times 3$ pixels for Sounder representing $30 \times 30 \mathrm{~km}$. Initially, a single clear-sky Imager radiance, $\tilde{R}_{\text {clear }(i m g)}$ is determined corresponding to every Sounder FOR by taking the maximum brightness temperature among the collocated Imager pixels (7x7 pixels) inside each FOR. This maximum brightness temperature is then converted to the radiance using Planck's function. The conceptual diagram is shown in figure 3. A relationship has been established using collocated Imager and Sounder window channel radiances which is used to convert Imager window clearsky radiance to equivalent clear-sky window channel Sounder radiance. This relationship is given by

$$
\tilde{R}_{\text {clear }(\text { snd })}=a \cdot \tilde{R}_{\text {clear }(\mathrm{img})}+b
$$

Where $a$ and $b$ are constants that are chosen dynamically by fitting the Imager window channel radiance with that of Sounder.

This $\tilde{R}_{\text {clear(snd) }}$ is considered as the required clear sky radiance, $\tilde{R}_{\text {clear }}$ in equation (4). $N^{*}$ is determined by measuring the fractional cloud cover between nearest pixels assuming the single cloud type under this study for INSAT3D Sounder. In order to make this process most effective, adjacent 8 pixels have been used to calculate $N^{*}$ and so cloud-cleared radiances consequently for a single FOV under consideration. The optimal clear radiance was chosen from least square method to make it stochastically significant. 


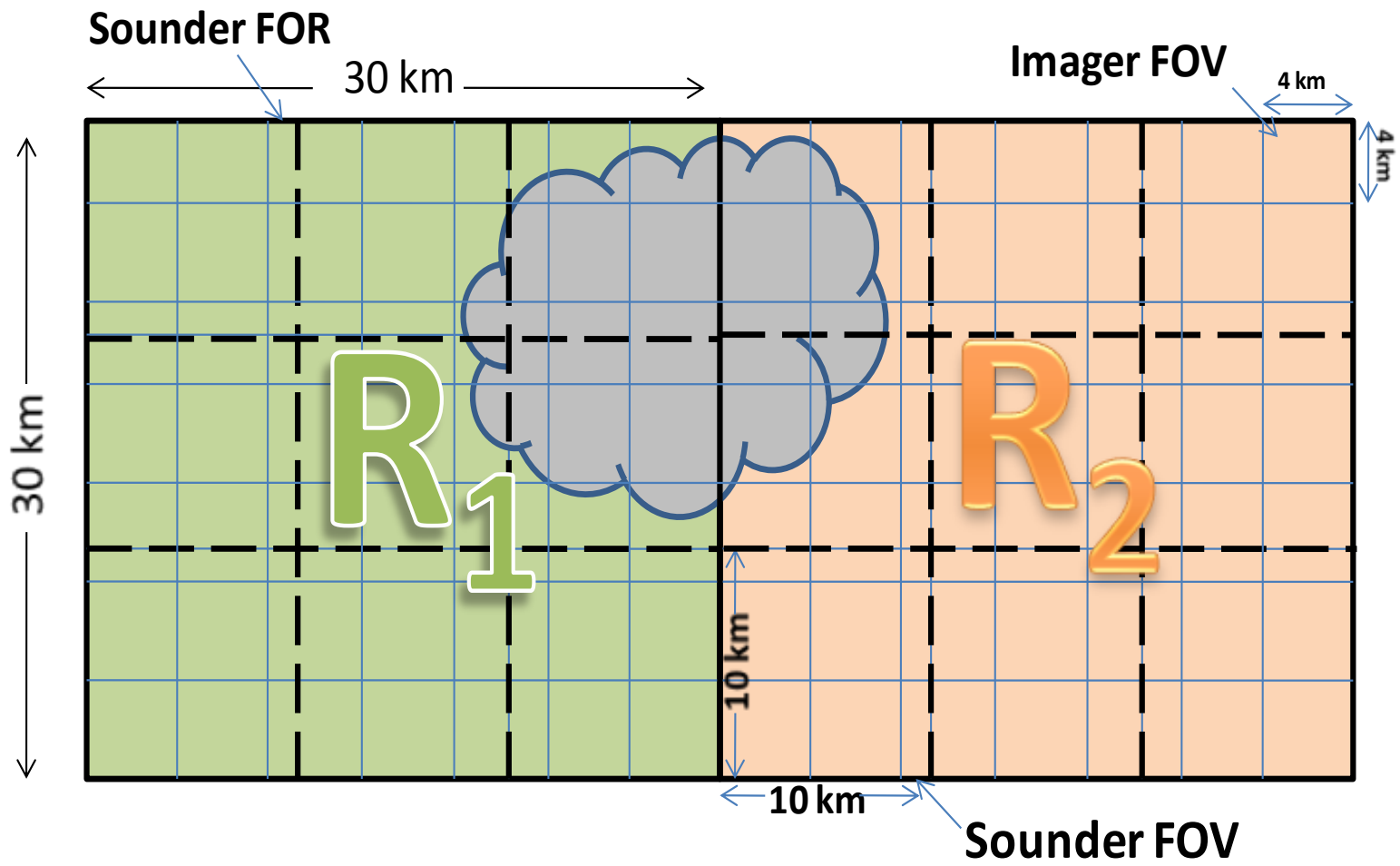

Figure 3: Pictorial representation of cloud clearing mechanism

Nevertheless, this method has a limitation that the adjacent pixels being considered must have variable amount of cloud fractions. Cloud-clearing is not attempted for the pair of sounder pixels that has same amount of cloud cover, or are either completely cloudy or clear.

The validation of the current approach of cloud-clearing is carried out using the operational cloud-cleared radiances available in AIRS level-2 products [6]. These cloud-cleared radiances are derived using a state-of-the-art technique that utilizes the collocated microwave sounder observations [7]. INSAT-3D Sounder equivalent cloud cleared radiance from

\subsection{Results and discussions:}

An apparent comparison of difference of INSAT-3D Sounder L1B radiance (INS3D_RAD) with its cloudcleared radiance (INS3D_CCR) and the difference with convolved AIRS L2 operational cloud-cleared radiance (INS3D_AIRS_CCR) for its thermal IR channel for four different cases is combined altogether and has been shown in figure 4. The results obtained are encouraging and ensures that the synergistic use of Imager radiances can help in determining the cloud-cleared radiances of Sounder channels. The discrepancies observed may be attributed to
AIRS cloud cleared radiance is computed by convolving over INSAT-3D SRFs using following formula:

$\operatorname{INS} 3$ D_AIRS_CCR $(i)=\frac{\sum A I R S \_C C R(i) \times S(i)}{\sum S(i)}$

Where, INS3D_AIRS_CCR is convolved INSAT-3D Sounder radiance, $S_{\lambda}$ is SRF for INSAT-3D Sounder, and AIRS_CCR is AIRS cloud-cleared radiance, and index ' $i$ ' represent channel number.

the assumption of single type of cloud in the FOR, and partly to the geo-location error in both the sensors. This may also be due to the fact that even Imager observations at $4 \mathrm{~km}$ resolution may have poor probability of finding clear pixel that may require spatial resolution of $1 \mathrm{~km}$ or better. Further, the assumption that the atmosphere is homogenous within $30 \mathrm{~km}$ FOR may not be valid over many regions, especially over land regions. Nevertheless, use of cloudclearing process may lead to increase number of retrieval yield. 


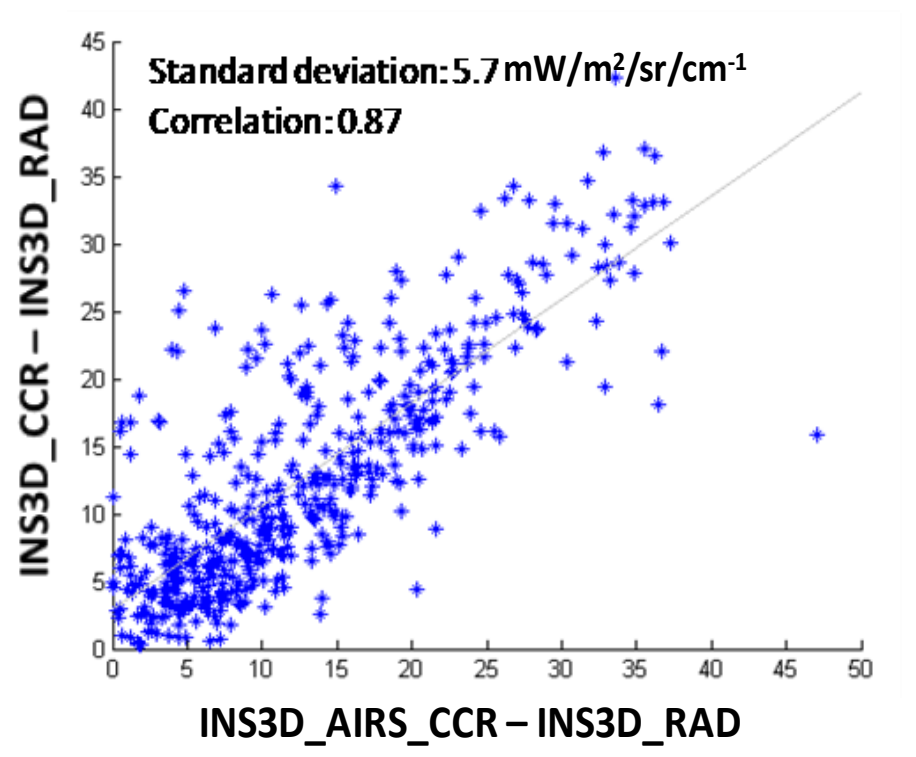

Figure 4: Scatter plot of difference of cloud clear radiance and L1 radiance of INSAT-3D Sounder with Imager compared to that of AIRS L2 operational cloud clear radiance

\section{CONCLUSION}

Annual Probability of clear sky is calculated from 4 monthly averages which are taken for every 3rd day by using MODIS confident clear pixels in Indian region for 3 different FOVs of 2,4,10 km inside 3 different FORs (10, $30,50 \mathrm{~km})$. Probability is calculated by counting the chance of ' $n$ ' $(=1,2,4,8,16,32$, or all $)$ clear FOVs in each FOR for three different confidence levels (100\%, 75\%, $50 \%$ ) of clear FOVs by counting MODIS clear pixels within it. Significant improvement in the probability has been observed as the FOV size decreases ranging from $10 \%$ to $50 \%$ corresponding to the spatial resolution reduction from $10 \mathrm{~km}$ to $2 \mathrm{~km}$.

Hence cloud-clearing is necessary requisite for each FOV at $10 \mathrm{~km}$ resolution, whereas, the same may not be required for FOV of $2 \mathrm{~km}$ size. In addition to this, a larger FOR by considering averaged FOVs provides better result than the individual FOVs. This work gives a clear picture of requirement of cloud-clearing for coarser observation as the probability of finding clear-sky is to be very less. However, for finer resolution observations, the enhanced probability of clear-sky region implicates the importance of employment of such high resolution Sounder/Imager for meteorological purpose.

Finally, a simple method of cloud-clearing for INSAT-3D Sounder using simultaneous observations from high resolution Imager has been attempted. It has shown a promising way to compute cloud-cleared radiances. The standard deviation of $5.7 \mathrm{~mW} / \mathrm{m}^{2} / \mathrm{sr} / \mathrm{cm}^{-1}$ with a correlation coefficient of 0.87 gives satisfactory results when validated with AIRS L2 operational cloud-cleared products.

\section{Reference:}

[1] R. Rizzi, C. Serio, G. Kelly, V. Tramutoli, A.MacNally, V. Kuomo; J. App. Meteorol;1994,33,179-194.

[2] Jun Li, Chian-Yi Liu, Hung-Lung Huang, Timothy J. Schmit, Xuebao Wu, W. Paul Menzel, and James J. Gurka; IEEE Transactions on Geoscience and Remote Sensing; 2005; 43, 6, 1266-1278.

[3] E. G. Pavelin, S. J. English and J. R. Eyre; Q. J. R. Meteorol. Soc.134: 737-749 (2008)

[4] Steve Ackerman, Kathleen Strabala, Paul Menzel, Richard Frey, Chris Moeller, Liam Gumley, Bryan Baum, Suzanne Wetzel Seeman and Hong Zhang; Discriminating clear-sky from cloud with modis algorithm theoretical basis document (mod35);NOAA.

[5] Jun Li, W. Paul Menzel, Zhongdong Yang, Richard A. Frey and Steven A. Ackerman; High-Spatial-Resolution Surface and Cloud-Type Classification from MODIS Multispectral Band Measurements; 2004, 43, 1619-1634.

[6] Edward T. Olsen, AIRS/AMSU/HSB Version 5 Data Release User Guide.

[7] Chris Barnet; Remote Sounding Notes; NOAA; 2007, 196-228. 